\title{
IMMUNOHISTOCHEMICAL MARKERS OF CLINICAL SIGNIFICANCE IN CARCINOMA OF THE COLON AND RECTUM
}

\author{
Renuka Patil ${ }^{1}$, Shanthala P. R2, Prema Saldanha ${ }^{3}$ \\ ${ }_{1}^{1}$ Postgraduate, Department of Pathology, Yenepoya Medical College, Yenepoya University, Mangaluru. \\ ${ }^{2}$ Associate Professor, Department of Pathology, Yenepoya Medical College, Yenepoya University, Mangaluru. \\ 3Professor, Department of Pathology, Yenepoya Medical College, Yenepoya University, Mangaluru.
}

\begin{tabular}{l} 
ABSTRACT \\
\hline BACKGROUND \\
Ki-67, a proliferative cell marker has a potential to distinguish rapidly proliferative tumours that are more likely to respond to \\
chemotherapy. The Her2/neu is a marker of prognostic and therapeutic significance that has been in clinical use for breast \\
carcinomas. Similar studies related to other tumours like colorectal carcinomas are needed to explore the significance. \\
The aim of the present study was to analyse the expression of Ki- 67 and Her2/neu status in colorectal carcinomas in correlation \\
with parameters like histological type, grade, tumour size and lymph node status.
\end{tabular}

\section{MATERIALS AND METHODS}

The retrospective study included 30 histologically diagnosed cases of colorectal carcinomas. The clinical and pathological findings were recorded. Immunohistochemical study was performed with Ki-67 and Her2/neu markers on all cases and the findings were independently interpreted by two observers.

\section{RESULTS}

The cases were in the age range of $30-75 \mathrm{yrs}$. with a M:F ratio of $1.2: 1$. Most of the tumours were in the left colon with rectum being the commonest site. Adenocarcinoma, NOS was the commonest type (86\%). High Ki-67 labelling (>50\%) was seen in 18 cases (60\%) of adenocarcinomas. The Her2/neu positivity was observed only in 3 cases of grade I adenocarcinomas.

\section{CONCLUSION}

The Ki-67 marker study would precisely define the cases with higher proliferative rate. Their predictive role could be of potential use for individualisation of adjuvant therapy. Her2/neu expression is low in colorectal carcinomas and need larger studies with combination of IHC and molecular methods along with clinical approach to determine the possible use in targeted therapy for colorectal carcinomas.

\section{KEYWORDS}

Colorectal Carcinoma, Ki-67, Her2/neu.

HOW TO CITE THIS ARTICLE: Patil R, Shanthala PR, Saldanha P. Immunohistochemical markers of clinical significance in carcinoma of the colon and rectum. J. Evolution Med. Dent. Sci. 2016;5(96):7087-7090, DOI: 10.14260/jemds/2016/1603

\section{BACKGROUND}

Colorectal carcinomas account for about $9.7 \%$ of all cancers and are the fourth leading cause of cancer related mortality in men. ${ }^{1}$ Colorectal carcinomas occur either sporadically (85\%) or as a part of hereditary cancer syndromes $(<10 \%)$. Colorectal carcinogenesis involves a multistep process of molecular genetic alterations. The biological behaviour of these carcinomas are variable and are mostly assessed based on morphological factors of prognostic importance. ${ }^{2}$

Presently, biomarkers of prognostic and therapeutic significance are being explored to improve the clinical outcome in these tumours. The rate of proliferation of these carcinomas is directly related to the aggressive behaviour and prognosis. However, on the other hand the rapidly

Financial or Other, Competing Interest: None.

Submission 26-10-2016, Peer Review 16-11-2016,

Acceptance 25-11-2016, Published 01-12-2016.

Corresponding Author:

Dr. Renuka Patil,

Postgraduate,

Department of Pathology,

Yenepoya Medical College,

Yenepoya University, Mangaluru-575018.

E-mail:renu83r@gmail.com

DOI: $10.14260 /$ jemds/2016/1603 proliferating tumours have been found to respond well to chemotherapy. ${ }^{3} \mathrm{Ki}-67$ is a proliferation associated nuclear protein that has been studied to assess the proliferative rate. This marker is found to be expressed during all phases of the cell cycle except in the resting phase. ${ }^{4}$ The guidelines of Her2/neu testing in breast carcinomas for adjuvant therapy is well established. Similar significance has also been found in gastric carcinomas. ${ }^{5,6}$ Thus, the present study was proposed to explore the Ki-67 and Her2/neu expression status in colorectal carcinomas in correlation with clinicopathological features.

\section{MATERIALS AND METHODS}

A retrospective study was done on 30 histopathologically diagnosed cases of colorectal carcinomas. Clinical data were recorded from the files. The $\mathrm{H}$ and $\mathrm{E}$ slides were retrieved from the archives of the Department of Pathology and histomorphological features were analysed. Tumours were classified and graded from I to III according to the current WHO classification. ${ }^{1}$ The depth of invasion of tumour on microscopic examination was considered as $\mathrm{T}$ category with reference to current WHO.

One paraffin-embedded block was selected and standard 4 $\mu \mathrm{m}$ sections were subjected to Immunohistochemical (IHC) study. Primary antibody to Ki-67 (Mouse Monoclonal 
Antibody, Pathnsitu) and antibody to Her2/neu (Rabbit Monoclonal Antibody, Pathnsitu) were used. The Polyexcel HRP (Non-biotin, micropolymer based) DAB Detection system were used with adequate positive and negative controls.

\section{Interpretation of IHC Results}

Ki-67 was recorded as positive in tumour cells as identified by presence of brown-coloured product in the nucleus. Ten High Magnification Fields (HPF) were observed of approximately 100 cells in each field. The average of positive cells in $10 \mathrm{HPF}$ was expressed as percentage of positive cells. In biopsies, the percentage of cells positive for 100 cells was assessed and expressed as percentage. The percentage of positive cells were scored as $<50 \%$ and $>50 \%$. Groups of cells showing $>50 \%$ expression were classified as high proliferative groups, while those showing $<50 \%$ expression were classified as low proliferative groups.

Her2/neu staining was scored as $0=$ no reactivity or membranous reactivity in $<10 \%$ of the tumour cells; $1+=$ faint or barely perceptible membrane staining of $>10 \%$ of tumour cells; $2+=$ weak-to-moderate complete, basolateral or lateral membrane staining of $>10 \%$ of tumour cells; $3+=$ strong complete, basolateral or lateral membrane staining of $>10 \%$ of tumour cells. Scores of 0 or $1+$ was considered tumour negative for Her2/neu expression and scores of $2+$ and $3+$ were regarded as positive expression of Her2/neu. ${ }^{6}$

The slides were observed and scored by two independent observers with consensus reached in any discrepancy.

\section{RESULTS}

The present study included total 30 cases of colorectal carcinomas. The patients were in the age range of 20 - 75 years with mean age being 54.43 yrs. The gender distribution showed male predominance, 18 cases $(60 \%)$ with a M:F ratio of 1.2:1. The left-sided colon tumours were common with 28 cases with rectum being the commonest site (rectum - 16 cases). There were only 2 cases of right-sided colon (ascending colon) tumours. Based on the tumour size, they were divided into two categories, those with average diameter of $<5 \mathrm{~cm}(15$ cases) and $\geq 5 \mathrm{~cm}$ (15 cases). Of the histological types, the commonest type was adenocarcinoma (86\%). There were 2 cases of mucinous carcinoma and 1 case each of signet ring and small cell carcinoma. Among the adenocarcinoma cases, the number of cases in each group were Grade I - 17 cases (65\%), Grade II - 8 cases (31\%) and Grade III - 1 case (4\%). The primary tumour category and lymph node status was available in excision specimens (20 cases). The commonest primary tumour categories ( $\mathrm{T}$ ) of the tumours were T3 (55\%). The lymph node positive status were observed in 6 cases (30\%).

The study of Ki-67 expression revealed (high expression > $50 \%$ ) (Figure 1) in 18 cases (60\%) of colorectal carcinomas. All the 4 cases of other histological types showed high expression (Figures 1B, C and D). In Grade I, tumours the Ki67 expression was high in $53 \%$ of cases. The Grade II tumours showed equal distribution (50\%) across the low and high expression groups. There was only one case of Grade III showing high expression. On comparison, Ki-67 expression in the groups based on tumour size in $<5 \mathrm{cms}$ size group $60 \%$ cases showed high expression, whereas in the $\geq 5 \mathrm{cms}$ size group $47 \%$ of the tumours showed high expression. On comparison of lymph node status and Ki-67 expression 40\% cases of positive group and $64 \%$ of cases of negative group showed high expression. On comparison of tumour category, $64 \%$ of $\mathrm{T} 3$ tumours showed high expression.

Her2/neu expression was positive only in 3 cases $(10 \%)$ of Grade I adenocarcinoma NOS type with score of $2+$ in all (Figure 2A). However, some of the adenocarcinomas ( 9 cases, $30 \%$ ) showed cytoplasmic staining of variable intensity which was considered as negative. The Her2/neu expression were seen in 1 case of $<5 \mathrm{~cm}$ size group and 2 cases of the $\geq 5 \mathrm{~cm}$ group. In relation to lymph node status, 2 cases were seen in the positive group and 1 case in the negative group. The tumour categories ( $\mathrm{T}$ ) of these three cases were $\mathrm{T} 3$. The results of the immunohistochemical study and correlation with the pathological parameters like histologic type, grade, tumour size, T category and lymph node status are shown in Table 1.

\begin{tabular}{|c|c|c|c|c|}
\hline & Ki-67 (<50\%) & Ki-67 (> 50\%) & Her2/neu Positive & Her2/neu Negative \\
\hline \multicolumn{5}{|l|}{ Histological Type } \\
\hline Adenocarcinoma $(n=26)$ & 12 & 14 & 3 & 23 \\
\hline Mucinous $(\mathrm{n}=2)$ & - & 2 & - & 2 \\
\hline Signet Ring cell $(n=1)$ & - & 1 & - & 1 \\
\hline Small Cell $(\mathrm{n}=1)$ & - & 1 & - & 1 \\
\hline \multicolumn{5}{|l|}{ Grade } \\
\hline Grade I (n=17) & 8 & 9 & 3 & 14 \\
\hline Grade II $(\mathrm{n}=8)$ & 4 & 4 & - & 8 \\
\hline Grade III $(n=1)$ & - & 1 & - & 1 \\
\hline \multicolumn{5}{|l|}{ Tumour size } \\
\hline$<5 \mathrm{~cm}(\mathrm{n}=15)$ & 6 & 9 & 1 & 14 \\
\hline$\geq 5 \mathrm{~cm}(\mathrm{n}=15)$ & 8 & 7 & 2 & 13 \\
\hline \multicolumn{5}{|l|}{ Depth of Tumour Invasion } \\
\hline $\mathrm{T} 1(\mathrm{n}=2)$ & 1 & 1 & - & 2 \\
\hline $\mathrm{T} 2(\mathrm{n}=7)$ & 4 & 3 & - & 7 \\
\hline $\mathrm{T} 3(\mathrm{n}=11)$ & 4 & 7 & 3 & 8 \\
\hline \multicolumn{5}{|l|}{ Lymph Node Status } \\
\hline Positive $(n=6)$ & 4 & 2 & 2 & 4 \\
\hline Negative $(n=14)$ & 5 & 9 & 1 & 13 \\
\hline \multicolumn{5}{|c|}{$\begin{array}{l}\text { Table 1. Immunohistochemical Expression of Ki-67 and } \\
\text { Her2/Neu in Correlation with Pathological Parameters }\end{array}$} \\
\hline
\end{tabular}




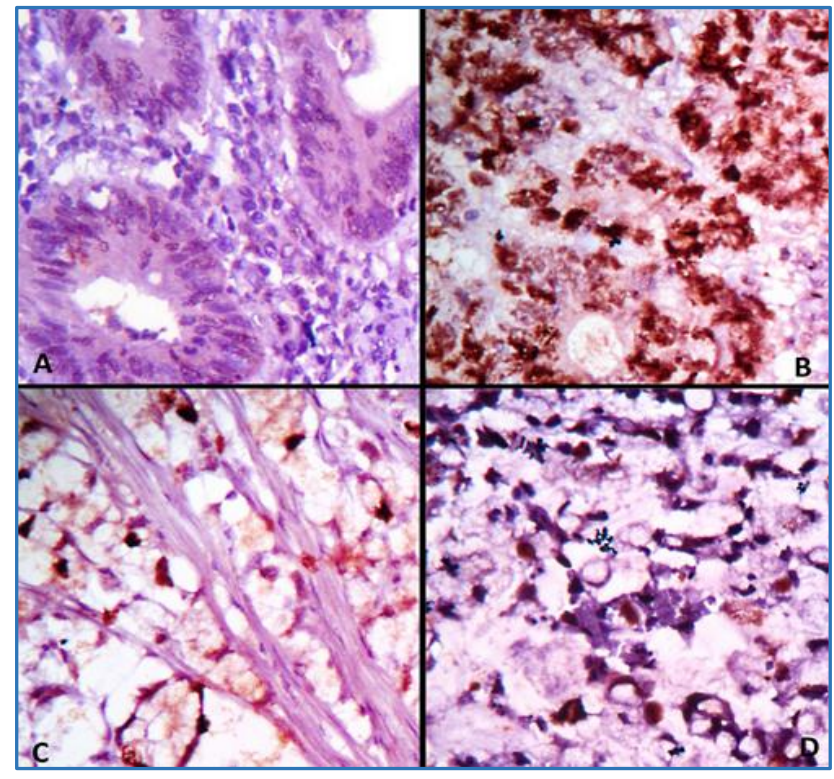

Figure 1. $A, B, C, D$

Figure 1. (A) Photomicrograph of Ki-67 Immunostain in Well-Differentiated Adenocarcinoma showing $<50 \%$ Expression, Magnification $\times 40$; (B) Photomicrograph of Ki67 Immunostain in Well-Differentiated Adenocarcinoma showing $>50 \%$ Expression, Magnification $\times 40$; (C)

Photomicrograph of Ki-67 Immunostain in Mucinous Adenocarcinoma showing $>50 \%$ Expression,

Magnification ×40; (D) Photomicrograph of Ki-67 Immunostain in Signet Ring Cell Carcinoma showing $>50 \%$ Expression, Magnification $\times 40$

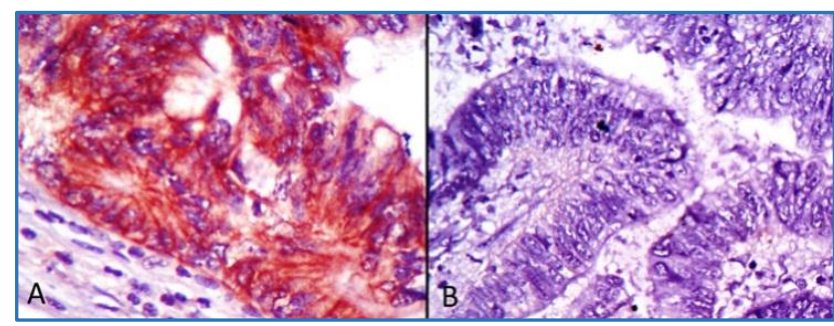

Figure 2. (A) Photomicrograph of Her2/Neu Immunostain in Well-Differentiated Adenocarcinoma showing (2+) Expression, Magnification ×40; (B) Photomicrograph of Her2/Neu Immunostain in Well-Differentiated Adenocarcinoma showing Negative (0) Expression, Magnification $\times 40$

\section{DISCUSSION}

The incidence of colorectal carcinomas has shown an increasing trend in the past few years with diet, lifestyle and genetic factors playing a key role in carcinogenesis. Most of the Indian studies reveal the age range of occurrence of these tumours as 45 - 84 years with a male predominance. ${ }^{7,8}$ The rectum and sigmoid colon are the commonest sites of carcinomas, which is in concordance with the present study and that of others. ${ }^{7,9}$ Various studies show adenocarcinoma, usual type as most common type (66 - 91\%), 2,7 and Grade II as the commonest grade.2,8 The morbidity and mortality associated with colorectal tumours has been an important concern in patient care. The constant study and exploration of biomarkers and therapeutic targets are needed to bring about better management protocols. In the present study, biomarkers of clinical interest like Ki-67 and Her-2/neu were studied.
Sustained cellular proliferation is an important feature acquired during the process of tumour genesis. Ki-67 is a proliferative cell marker, which is identified as a nuclear protein expressed during all active phases of the cell cycle except in the G0 phase. Ki-67 labelling index is usually studied for its predictive and prognostic value. However, most of the studies done have used different methods of assessment, making comparison of studies difficult. In colorectal carcinomas, the cut-off values for categorisation into low and high proliferation tumours has not been in consensus. ${ }^{3,4}$ In the present study, the cut-off value of $50 \%$ of positive cells was used for categorisation as most studies found 40 - 60\% cut-off to be of prognostic significance..$^{3,4}$

The associations between Ki-67 Proliferative Index (PI) and various prognostic factors like tumour size, histological type, grade and stage of the tumour have been studied with assumed direct relationship. Interestingly, the results of these have been variable as also observed in our study. In one of the study, the mean Ki-67 PI was highest in mucinous carcinomas and lowest in usual type adenocarcinomas, ${ }^{7}$ whereas another study showed a high proliferative index in non-mucinous (Adenocarcinoma NOS type). ${ }^{2}$ In our study, the number of mucinous carcinomas was limited. In relation to the grade, some studies identified that the Ki- 67 index was proportional to the grade of tumour, 7,8 whereas another study showed high index in Grade I tumours. ${ }^{8}$ In our study, Ki-67 expression was variable and not proportional to the grades of the tumours. Though in some of the tumours the Ki-67 index has also been used as criteria to grade the tumours, the same may not be of significance in colorectal carcinomas.

The expression of Ki-67 has been heterogeneous in relation to the pathological parameters. In the current study, both $<5 \mathrm{~cm}$ and $>5 \mathrm{~cm}$ tumour size as well as lymph node status, either positive or negative showed equivocal expression with Ki-67. However, some of the low-grade carcinomas presented with higher stage of tumour (T category) and showed high expression with Ki-67. In the studies done correlating Ki-67 status and survival rates, it has been found that lower Ki-67 rate was associated with recurrence and lower patient survival. ${ }^{10}$ In contrast from the management view, the rapidly proliferating tumours have been found to respond well to adjuvant therapy. The Ki-67 assessment before therapy could predict the effectiveness of the treatment, whereas the same after treatment could predict the disease free survival of patients. ${ }^{11}$

Her2/neu oncogene, also known as c-erbB-2 located on the chromosome 17q21 and is similar to epidermal growth factor receptors Her1, Her3 and Her4 which encodes tyrosine kinase receptor family. Activation of Her2/neu will initiate signalling pathways like MAPK/PI3K/AKT, which is essential for cell proliferation and differentiation. ${ }^{12}$ Overexpression of Her2/ neu leads to unregulated cell growth and may drive oncogenic transformation. Her2/neu expression study have potential prognostic significance in addition to implication of therapeutic option with monoclonal antibodies. ${ }^{13}$

The Her2/neu protein overexpression or gene amplification is found to be associated with $25 \%$ of all gastrointestinal malignancies. ${ }^{12}$ The expression rate of Her2/ neu across various studies of colorectal carcinomas has been found to be in a range of $2.7 \%-47.7 \%$. The wide range of prevalence of Her2/neu expression rate is attributed to the discrepancy related to several factors like study group, sample 
size, different scoring systems followed and preanalytical factors (primary antibodies used and the IHC procedure). ${ }^{5,13}$

The Her2/neu expression scoring system introduced for breast carcinomas considered, $3+$ as positive and $2+$ as equivocal and 0 and $1+$ as negative. ${ }^{6}$ In Situ Hybridization (ISH) has been used to verify IHC-equivocal cases. Her2positive gastric cancer has been defined as IHC $3+$ or ISH positive in the USA and Japan and the FDA has approved trastuzumab in association with chemotherapy for metastatic gastric cancer limited to patients with a score of IHC $3+$ or $2+$ and ISH positivity. ${ }^{7}$ The completeness of membrane staining required for positivity in breast cancers is found to be infrequent in gastric adenocarcinomas, which often exhibit a basolateral staining pattern. Similar basolateral staining has also been observed in our study with colorectal carcinomas.

In context of colorectal carcinomas, the studies of Her2/neu expression, wherein the cytoplasmic staining was also considered as positive had a high frequency $(59 \%-65 \%)$ of positivity, ${ }^{14,15}$ whereas the studies considering only membrane positivity with a score $3+$ found that the expression was low (3\%). ${ }^{13}$ In a still larger study, ${ }^{5}$ only $0.5 \%$ cases (score $3+$ ) showed such positivity, but the gene amplification studies done on cases with equivocal score $(2+)$ revealed that $17 / 35$ cases were positive and all the score $3+$ cases were positive. Thus, in this CRC cohort study, $1.6 \%$ of cases showed Her2/neu positivity. 5 Hence, in our study we have considered both scores $2+$ and $3+$ as positive. However, in the present study there were only score $2+$ cases with none of (score $3+$ ).

The association between the Her2/neu status and pathological parameters is variable across the studies. A study had observed high expression in mucinous carcinomas (71.4\%) than conventional type (65\%) and 100\% expression in Grade 3 tumours and lymph node positive cases. ${ }^{15}$ In another study, high expression was observed in conventional adenocarcinoma type and Grade 2 tumours (85.7\%). A study found significant association of Her2/neu expression with higher stage, positive lymph node status and a tendency for poor overall survival. ${ }^{5}$ In contrast, another study observed reverse correlation of Her2/neu expression in relation to the grade and stage of the tumours. ${ }^{14}$ In our study all 3 positive cases were of adenocarcinoma NOS (Grade I) tumours, but had presented in higher primary tumour category (T3).

\section{CONCLUSION}

In the present study of colorectal carcinomas, the Ki-67 labelling was high (>50\%) in nearly about half of cases irrespective of pathological parameters, especially the grade and stage. However, the Ki-67 marker study would precisely define the cases with higher proliferative rate that are likely to respond well to chemotherapy. The predictive role could be of potential use for individualisation of adjuvant therapy. Her2/neu expression frequency was low in the present study and was mostly seen in adenocarcinomas of low grade, but of higher primary tumour category (T3). The study of Her2/neu expression needs larger studies with combination of IHC and molecular methods along with clinical approach to determine the possible use of targeted therapy in colorectal carcinomas.

\section{REFERENCES}

1. Bosman FT, Carneiro F, Hruban RH, et al. WHO classification of Tumours of the digestive system. World Health Organization classification of tumours. IARC Press, Lyon 2010:134-5.

2. Nabi U, Nagi AH, Sami W. Ki-67 proliferating index and histological grade, type and stage of colorectal carcinoma. journal of Ayub Medical College Abbottabad 2008;20(4):44-8.

3. Oshima CT, Iriya K, Forones NM. Ki-67 as a prognostic marker in colorectal cancer but not in gastric cancer. Neoplasma 2005;52(5):420-4.

4. Salminen E, Palmu S, Vahlberg T, et al. Increased proliferation activity measured by immunoreactive Ki67 is associated with survival improvement in rectal/recto sigmoid cancer. World Journal of Gastroenterology 2005;11(21):3245-9.

5. Heppner IB, Behrens HM, Balschun K, et al. HER2/neu testing in primary colorectal carcinoma. British Journal of Cancer 2014;111(10):1977-84.

6. Bang YJ, van Cutsem E, Feyereislova A, et al. Trastuzumab in combination with chemotherapy versus chemotherapy alone for treatment of HER2-positive advanced gastric or gastrooesophageal junction cancer (ToGA): a phase 3 , open-label, randomized controlled trial. Lancet 2010;376(9742):687-97.

7. Bhagyalakshmi A, Sreelekha A, Babu KA, et al. Ki-67 proliferation index and clinicopathological patterns in colorectal carcinomas. Journal of Clinical and Scientific Research 2015;4(2):119-28.

8. Sen A, Mitra S, Das RN, et al. Expression of CDX-2 and Ki67 in different grades of colorectal adenocarcinomas. Indian Journal of Pathology and Microbiology 2015;58(2):158-62.

9. Laishram RS, Kaiho N, Shimray R, et al. Histopathological evaluation of colorectal carcinoma status in Manipur, India. International Journal of Pathology 2010;8(1):5-8.

10. Reimers MS, Zeestraten ECM, Kuppen PJK, et al. Biomarkers in precision therapy in colorectal cancer. Gastroenterology Report (Oxf) 2013;1(3):166-83.

11. Fodor IK, Hutchins GG, Espiritu C, et al. Prognostic and predictive significance of proliferation in 867 colorectal cancers. journal of clinical pathology 2012;65(11):98995.

12. Farzand S, Siddique $\mathrm{T}$, Saba $\mathrm{K}$, et al. Frequency of HER2/neu overexpression in adenocarcinoma of the gastrointestinal system. World Journal of Gastroenterology 2014;20(19):5889-96.

13. Schuell B, Gruenberger T, Scheithauer W, et al. Her-2/neu protein expression in colorectal cancer. Bio Med Center Cancer 2006;6:123.

14. Ghaffarzadegan K, Sharifi N, Vosooghynia H, et al. Her$2 /$ neu expression in colon adenocarcinoma and its correlation with clinicopathologic variables. International journal of basic medical science 2006;1:64-69.

15. Gill MK, Jain K, Manjari M, et al. Expression of Her-2/neu in colon carcinoma and its correlation with the histological grades and the lymph nodes status. Journal of Clinical and Diagnostic Research 2011;5(8):1564-68. 